\title{
THE UNITY IN RINGS WITH GABRIEL AND KRULL DIMENSION
}

\author{
F. HANSEN
}

\begin{abstract}
The main result is that all rings with right Krull dimension and divisible torsion free additive group have a right identity. Furthermore it will be proved that a simple ring with characteristic 0 , right Gabriel dimension $<2$ and finite right uniform dimension has a unity. This is false for higher Gabriel dimensions, as demonstrated by a counterexample. A similar construction gives an example for a ring with unity and Gabriel dimension, but without Krull dimension, all factor rings having finite uniform dimension.
\end{abstract}

1. Introduction. In (8) it was shown that every simple right Noetherian ring $R$ with $\operatorname{char}(R)=0$ contains a unity. This was generalized in two directions: All right Noetherian rings with divisible additive group and all simple rings $R$ with $\operatorname{char}(R)=0$ and right Krull dimension have an identity (see [5] and [10]). Our main aim is to prove the following theorem covering the results mentioned above: Every ring with divisible torsion free additive group and right Krull dimension has a right unity (Theorem 3.3).

The proof of Theorem 3.3 needs that every ring $R$ with $x \in x R$ for all $x \in R$ and right or left Krull dimension has a right unity (Proposition 1.3). From this we also get the following equivalent conditions (Corollary 2.3): (a) $R$ has a right unity and is right Noetherian; (b) the polynomial ring $R[x]$ in the commuting indeterminate $x$ is right Noetherian; (c) $R[x]$ has right Krull dimension. The first equivalence has been shown in [2] for commutative rings.

In $\S 4$ it will be proved: Let $R$ be a simple ring with right Gabriel dimension $\leqslant 2$ and characteristic 0 . Then $R$ is right Goldie if and only if it contains a unity (Corollary 2.4). This is not true for rings with Gabriel dimension $>2$, as is shown by the construction of a simple right and left Ore domain with characteristic 0 and right Gabriel dimension 3, but without unity.

The results have some consequences for rings with identity: Let $D$ be any domain with identity which is an algebra over a field with characteristic 0 . Then by Theorem 3.3 no proper right or left ideal $L$ of $D$ can have Krull dimension qua ring, even if $D$ has. On the other hand, the example above is a ring of type $L$. Consider the ring (with unity) $L_{1}=\mathbf{Z}+L$ which likewise has Gabriel dimension but no Krull dimension (compare \$2). We will see that each factor ring of $L_{1}$ has finite right uniform dimension. Thus $L_{1}$ verifies a conjecture in $[3$, p. 271] on the existence of such rings (it should be remarked that they share numerous properties with rings which have Krull dimension,

Received by the editors February 7, 1975 and, in revised form, July 11, 1975.

AMS (MOS) subject classifications (1970). Primary 16A32, 16A46.

Key words and phrases. Right unity, unity, Krull dimension, Gabriel dimension, simple ring, Goldie ring, maximum condition for annihilating right ideals, finite uniform dimension. 
e.g. each ideal $I$ contains a product of prime ideals $\supseteq I$ ).

Conditions for the existence of a right identity like that in Proposition 4.4 or that cited before can be used to prove decomposition theorems on (weakly) regular rings, in particular $V$-rings (see [6]).

2. Krull and Gabriel dimensions: basic properties. At first let us summarize the properties we need. They all can be found in [3] or [4], though some were known before. We omit the definitions of Gabriel and Krull dimension of an $R$-module $M_{R}$, denoted by $G\left(M_{R}\right)$ and $K\left(M_{R}\right)$. Let us solely remark that $G\left(M_{R}\right)=0$ stands for $M_{R}=(0)$ and $G\left(M_{R}\right)=1$ means $M_{R}$ is semi-Artinian, i.e. every nonzero factor module contains a simple submodule; $K\left(M_{R}\right)=0$ means $M_{R}$ is Artinian.

In [3] these concepts are only defined for rings with unity and unitary modules, but it is not hard to see that a unity in the definition is superfluous. Moreover one can reduce the definition to rings with identity by: $G\left(M_{R}\right)=\beta$ iff $G\left(M_{R_{1}}\right)=\beta, \beta$ some ordinal number and $R_{1}$ the usual overring of $R$ with unity; the same for $K\left(M_{R}\right)$.

1.2. The class of all $R$-modules $M_{R}$ with $G\left(M_{R}\right) \leqslant \beta$ is an hereditary torsion class. In contrast to this the class of all modules $M_{R}$ with $K\left(M_{R}\right) \leqslant \beta$ is closed under group extensions, submodules and factor modules.

2.2. A module has Krull dimension if and only if it has Gabriel dimension and all of its factor modules have finite uniform dimension. A module is said to have finite uniform dimension if it contains no infinite direct sum of submodules.

3.2. If $K\left(M_{R}\right)$ is finite, $G\left(M_{R}\right)=K\left(M_{R}\right)+1$.

4.2. Let $P(R)$ be the prime radical of a ring $R$. If $R$ has Gabriel dimension and all factor rings of $R$ have finite right uniform dimension, then $R / P(R)$ is right Goldie and $P(R)$ is nilpotent. In particular this is valid if $R$ has right Krull dimension.

5.2. If $R[x]$ has right Krull dimension, $R$ is right Noetherian.

6.2. A semiprime ring $R$ with right Gabriel dimension and finite right uniform dimension has the following properties: (a) $R$ is right Goldie, (b) $G(R / E)<G\left(R_{R}\right)$ for every essential right ideal $E$ of $R$.

7.2. A simple ring (only with unity !) and right Gabriel dimension is right Goldie.

8.2. An $R$-module $M_{R}$, each of its proper factor modules having Gabriel dimension $\leqslant \beta$, has Gabriel dimension $\leqslant \beta+1 ; G\left(M_{R}\right)=\beta+1$, if $G\left(M_{R}\right) \neq \beta$.

9.2. Every Noetherian module has Krull dimension.

10.2. The ring $\mathbf{Z}$ has Krull dimension 1; hence by 3.2 each $\mathbf{Z}$-module has Gabriel dimension $\leqslant 2$.

11.2. It is generally known that every (right and left) hereditary Noetherian prime ring (with unity) has right and left Krull dimension 1.

Clearly 1.2 to 3.2 and $8.2,9.2$ remain true for nonunitary modules (it can be shown with the aid of $R_{1}$ ). An exact study of the proofs makes clear that in 4.2 to 6.2 the unity likewise is no condition (in some cases it also can be proved via $R_{1}$ ). 7.2 fails for rings without unity, because the ring $R$ of all linear transformations of finite rank over an infinite dimensional vector space is a non-Goldie simple ring without unity, but $R$ is generated by its simple 
submodules, hence has Gabriel dimension 1. The question whether $R$ in 5.2 must have an identity is just the tenor of Corollary 2.3.

3. The unity in rings with Krull dimension. Since every right Noetherian ring has right Krull dimension, the following proposition is a generalization of the same result for right and left Noetherian rings in [5]. The proof is essentially the same.

Proposition 1.3. Every ring $R$ with $x \in x R$ for all $x \in R$ and right or left Krull dimension contains a right unity.

Proof. According to $4.2 P(R)$ has a degree of nilpotency, say $n$. In case $n=1, R$ is a semiprime right or left Goldie ring by 4.2 ; hence there exists a regular element $x=x r ; r$ is a left unity, because $r s=s$ for all $s \in R$ is equivalent to $x r s=x s$ being true. As $R$ is semiprime, $r$ is a unity.

Next let $n>1 ! R / P(R)^{n-1}$ is a ring satisfying the same conditions as $R$ and thus, by induction, contains a right unity $e+P(R)^{n-1} ; e$ can be chosen idempotent, since idempotents modulo the prime radical can be lifted to idempotents of $R$. Furthermore

$$
P(R)=P(R) R=P(R)\left(R e+P(R)^{n-1}\right)=P(R) e,
$$

so

$$
R=P(R)^{n-1}+R e \subseteq P(R)+R e=R e .
$$

An idempotent element $e$ with $R e=R$ is a right unity.

In view of 4.2 the proposition can be generalized for rings with right (left) Gabriel dimension, all factor rings having finite right (left) uniform dimension.

COROLlaRY 2.3. For a ring $R$ the following conditions are equivalent:

(a) $R$ is right Noetherian and has a right unity.

(b) $R[x]$ has right Krull dimension.

(c) $R[x]$ is right Noetherian.

Proof. (a) $\Rightarrow$ (c). Hilbert basis theorem.

(c) $\Rightarrow$ (b). A priori.

(b) $\Rightarrow$ (a). With regard to 5.2 it only must be shown that $R$ has a right unity: Let $r \in R$ and $K=\sum_{i=0}^{\infty} \mathbf{Z} r x^{i}$.

$L=(K+r R[x]) /(r R[x])$ is a trivial right $R[x]$-module and by 2.2 has finite uniform dimension being a submodule of $R[x] / r R[x]$. Consider $M=\bigoplus_{i=0}^{\infty}(\mathbf{Z} r+r R) / r R$. A group homomorphism from $K$ onto $M$ with kernel $r R[x] \cap K$ is defined by

$$
z_{0} r+z_{1} r x+\cdots+z_{n} r x^{n} \rightarrow\left(z_{0} r+r R, \ldots, z_{n} r+r R, 0,0, \ldots\right),
$$

$$
z_{i} \in \mathbf{Z}
$$

i.e. $L \cong M$, hence $r \in r R$. According to Proposition 1.3, $R$ has a right unity.

THEOREM 3.3. Let $R$ be a ring with right Krull dimension and divisible torsion free additive group. Then $R$ has a right unity.

Proof. For each $x \in R, x R$ is a divisible subgroup of $R$. Consider $K_{x}=\{r \in R ; r R \subseteq x R\}$; let $r \in K_{x}$ and $n$ any nonzero integer. There exists 
an $s \in R$ with $n s=r$; hence $x R \supseteq r R=n s R=s R$, i.e. $s \in K_{x}$. Thus $K_{x}$ is a divisible group and therefore $L_{x}=K_{x} / x R$, too. However $L_{x}$ is a trivial $R$-module and $\mathbf{Q}_{\mathbf{z}}$ has no Krull dimension, so $L_{x}$ must be a finite direct sum of quasicyclic groups $\mathbf{Z}\left(p_{i}^{\infty}\right), p_{i}$ a finite number of primes.

Now $L_{x}$ is Artinian; thus $M_{x}=(x \mathbf{Z}+x R) / x R$ also. This and $x \mathbf{Z} \cong \mathbf{Z}$ imply $x \mathbf{Z} \cap x R \neq(0)$. It follows from this that $x m=x r$ for some nonzero $m \in \mathbf{Z}, r \in R ; r$ is of the form $r=m s, s \in R$. Hence $x m=x m s$ and, since $R$ is torsion free, $x=x s$. Proposition 1.3 completes the proof.

COROLLARY 4.3. Every ring with divisible torsion free additive group and right and left Krull dimension has a unity.

COROLlary 5.3 [10]. Every nontrivial simple ring with characteristic 0 and right Krull dimension contains an identity.

Another consequence of Theorem 3.3 is that no proper right or left ideal of a domain $D$ with divisible torsion free additive group has Krull dimension qua ring, even if $D$ has. Such domains are ubiquitous amongst twisted polynomial rings and rings of differential polynomials. In contrast to this there exist Noetherian domains of prime characteristic, having proper right ideals, which are Noetherian qua rings (see [10]).

\section{The unity in rings with Gabriel dimension and in Goldie rings.}

Proposition 1.4. A semiprime ring $R$ with $G\left(R_{R}\right) \leqslant 2$, finite right uniform dimension and divisible additive group has a unity.

Proof. The additive group necessarily is torsion free, else it would contain $\mathbf{Z}\left(p^{\infty}\right)$, the quasicyclic group for some prime $p$. Let $x \in \mathbf{Z}\left(p^{\infty}\right)$ !. There is an $i$ such that $p^{i} x=0$. On the other hand for every $r \in R$ there is an $s \in R$ with $p^{i} s=r$. From this it follows that $\mathbf{Z}\left(p^{\infty}\right) R=0$, a contradiction to semiprime. In case $G\left(R_{R}\right)=1$, every factor module $\neq(0)$ of $R_{R}$ contains a simple submodule. By 6.2(a) $R$ is right Goldie. It is known that a semiprime right semi-Artinian right Goldie ring is Artinian, thus it contains a unity.

Let $G\left(R_{R}\right)=2$. By 6.2 (b) there is a regular element $x \in R$ with $G(R / x R)$ $=1$. Moreover $(x R+x \mathbf{Z}) / x R \cong x \mathbf{Z} / x R \cap x \mathbf{Z}$ is a trivial right $R$-module. Since $R$ is torsion free, $x \mathbf{Z} \cong \mathbf{Z} ; x R \cap x \mathbf{Z}$ must be different from zero, otherwise $(x R+x \mathbf{Z}) / x R$ would have no simple submodule. That is why $x e=x z$ for some nonzero $z \in \mathbf{Z}$ and an $e \in R$, and so $e r=z r$ for all $r \in R$; as $R$ is divisible, $z s=e$ for some $s \in R$, hence $e s=e$. Clearly $e$ is regular, thus $s$ is a left unity of $R$, and so a unity.

Corollary 2.4. Let $R$ be a simple ring with $\operatorname{char}(R)=0$ and $G\left(R_{R}\right) \leqslant 2$. Then $R$ is right Goldie if and only if it contains an identity.

Proof. 7.2 and Proposition 1.4.

Corollary 3.4. Let $R$ be a ring with divisible additive group, $G\left(R_{R}\right) \leqslant 2$ and $P(R) R=P(R)$ such that each factor ring has finite right uniform dimension. Then $R^{\circ}$ contains a right unity.

Proof. An inductive proof on the degree of nilpotency of $P(R)$. The first step is Proposition 1.4, the step $n-1 \rightarrow n$ is the same as in the proof of Proposition 1.3. 
Proposition 2.1 in [6] asserts that every ring with minimum condition for annihilating right ideals and $x \in x R$ for all $x \in R$ contains a right identity. It remains open whether the same is true for rings with maximum condition for annihilating right ideals. However we can show

Proposition 4.4. If $R$ is a right Goldie ring with $x \in x R$ for all $x \in R$, then $R$ has a right unity.

Proof. Let $Z(R)$ denote the right singular ideal of $R$. By [1, p. 512] $Z(R)$ is nilpotent if $R$ satisfies the maximum condition for annihilating right ideals. Furthermore, if $R$ has finite right uniform dimension, $R / Z(R)$ satisfies the minimum condition for annihilating right ideals according to [1, p. 511]. Because of the proposition in [6] $R / Z(R)$ has a right identity. An induction on the degree of nilpotency of $Z(R)$, similar to the proof of Proposition 1.3, completes the proof.

An example demonstrates that Corollary 2.4 and, in particular, Proposition 1.4 are false if $G\left(R_{R}\right)=3$ : Let $K$ be the field of rational functions over $Q$ and "'" the usual derivation on $K$. Further let $S=K[x$; '] be the ring of differential polynomials with coeffieicnts on the right, i.e. $k x=x k+k^{\prime}$, $k \in K . S$ is known to be a simple principal right and left ideal domain.

Now let $L \neq(0)$ be any proper right ideal of $S$. Clearly $L$ is a simple ring without unity. We first show that $L$ has right Gabriel dimension 3 qua ring, i.e. in accordance with 6.2 and 8.2 each proper factor module $F_{L}$ of $L_{L}$ has $G\left(F_{L}\right) \leqslant 2$ with $G\left(F_{L}\right)=2$ for some $F_{L}$. Let be $F_{L}=L / N$, where $N$ is a nonzero proper right ideal of $L . N S^{-1}=\{x \in N ; x S \subseteq N\}$ is the uniquely determined maximal $S$-submodule of $N . N S^{-1} \neq(0)$, because $S$ is a domain, therefore by $3.2,6.2$ and 11.2 there exists a simple $S$-module $C / N S^{-1} \subseteq$ $L / N S^{-1}(C \& N)$.

If $y L \subseteq N$ for some $y \in L \backslash N$, then $H=(y \mathbf{Z}+N) / N$ has Gabriel dimension $\leqslant 2$, since $H$ is a factor of $y \mathbf{Z} \cong \mathbf{Z}$. If alternatively $y L \subseteq N$ for all $y \in L \backslash N$, then $(C+N) / N$ is a simple $L$-module. To show this, let $c \in$ $(C+N) \backslash N ; c L \underline{\&} N S^{-1}$ implies $c L+N S^{-1}=C$, as $C / N S^{-1}$ is simple over $S$ and $\left(c L+N S^{-1}\right) / N S^{-1}$ is a nonzero $S$-submodule of $C / N S^{-1}$. Thus $c L+N=C+N$, whence $(C+N) / N$ is simple over $L$.

Now each proper factor module of $L_{L}$ has Gabriel dimension $\leqslant 2$, and so $G\left(L_{L}\right) \leqslant 3$ by 8.2 . Consider the right ideal $a L \neq(0)$ of $L, a \in L$, and the trivial $L$-module $K=(\mathbf{Z} a+a L) / a L$. From $a L$ torsion free and $a \notin a L$ follows $K \cong \mathbf{Z}$. Hence $G\left(K_{L}\right)=2$ and $G\left(L_{L}\right)=3$.

Let us remark that the proof is rather independent of the special structure of $S$. We only needed that $S$ is a simple right Ore domain with $\operatorname{char}(S)=0$ and $G\left(S_{S}\right)=2$.

It easily can be checked that all nonzero ideals of $\mathbf{Z}+L=L_{1}$ contain $L$. Of course $R$ and each factor ring of $R / L \cong \mathbf{Z}$ have finite right uniform dimension. $G\left(L_{1 L_{1}}\right)=3$ and the nonexistence of Krull dimension are immediate consequences of the same facts for $L_{L}$ (compare introduction).

\section{REFERENCES}

1. J. W. Fisher, Nil subrings with bounded indices of nilpotency, J. Algebra 19 (1971), 509-516. MR 44 \#6742.

2. R. W. Gilmer, Jr., If $R[X]$ is Noetherian, $R$ contains an identity, Amer. Math. Monthly 74 (1967), 700. MR 35 \#2882. 
3. R. Gordon, Gabriel and Krull Dimension, Ring Theory, Proc. Oklahoma Conference, 1974, pp. 241-295.

4. R. Gordon and J. C. Robson, Krull dimension, Mem. Amer. Math. Soc. No. 133 (1973).

5. F. Hansen, Die Existenz der Eins in noetherschen Ringen, Arch. Math. 25 (1974), 589-590.

6. __ On one-sided prime-ideals, Pacific J. Math. 58 (1975), 79-85.

7. B. Lemonnier, Sur l'existence d'un élément neutre dans certains anneaux, C. R. Acad. Sci. Paris Sér.A-B 268 (1969), A1519-A1522. MR 39 \#6924.

8. J. C. Robson, Do simple rings have unity elements? J. Algebra 7 (1967), 140-143. MR 35 \#1631.

9.

45 \#8687.

10. Simple noetherian rings need not have unity elements, Bull. London Math. Soc. 7 (1975), 269-270.

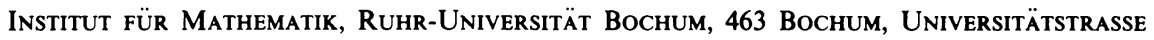
150, Bochum, Postfach 2148, Federal Republic of Germany 